2. L. H. Loomis, An introduction to abstract harmonic analysis, New York, Van Nostrand Company, 1953. 176.

3. J. D. Newburgh, The variation of spectra, Duke Math. J. vol. 18 (1951) pp. 165-

Purdue University

\title{
CONTINUITY PROPERTIES OF DERIVATIVES OF SEQUENCES OF FUNCTIONS ${ }^{1}$
}

\author{
G. R. MACLANE
}

In a recent paper Dvoretzky [1] discusses an interesting generalization of a theorem of Walsh [2]. A striking supplement to Dvoretzky's theorem is the following one.

THEOREM. There exists a sequence of functions

$$
\left\{f_{n}\right\}_{1}^{\infty}, f_{n} \in C^{1}(-\infty, \infty),
$$

with $\lim _{n \rightarrow \infty} f_{n}(x) \equiv 0$, such that: if $N_{1}$ is any subsequence of the natural numbers with the property that there exists a sequence $x_{n_{1}}, n_{1} \in N_{1}$, satisfying

$$
f_{n_{1}}^{\prime}\left(x_{n_{1}}\right)=0, \quad \text { and } \quad \lim _{n_{1} \rightarrow \infty} x_{n_{1}}=0,
$$

then the sequence $N_{2}$ complementary to $N_{1}$ (i.e., $N_{2}$ contains exactly those natural numbers omitted by $N_{1}$ ) is infinite and

$$
\limsup _{n_{2} \rightarrow \infty} \int_{0}^{h}\left|f_{n_{2}}^{\prime}(x)\right| d x=\infty
$$

for every $h>0$.

Proof. Let $\epsilon_{n} \downarrow 0$ and let $\left\{\lambda_{n}\right\}$ be the sequence $1,2,1,2,3,1,2,3$, $4,1, \cdots$. Let $\left\{\mu_{n}\right\}$ be a sequence of positive numbers such that

$$
\epsilon_{n} \mu_{n} / \lambda_{n} \rightarrow \infty,
$$$$
n \rightarrow \infty \text {. }
$$

The functions $f_{n}(x)$ shall be odd and

Received by the editors September 4, 1956.

1 This research was supported by the United States Air Force through the Air Force Office of Scientific Research of the Air Research and Development Command under contract No. AF 18(600)-1135. 


$$
f_{n}(x)=\left\{\begin{array}{lr}
\epsilon_{n} \sin \lambda_{n} x, & |x| \leqq \pi / 2 \lambda_{n}, \\
\epsilon_{n} \cos \mu_{n}\left(x-\frac{\pi}{2 \lambda_{n}}\right), & x>\pi / 2 \lambda_{n} .
\end{array}\right.
$$

It is easily verified that $f_{n} \in C^{1}$ and $f_{n}(x) \rightarrow 0$. The smallest zeros of $f_{n}^{\prime}(x)$ are $\pm \pi /\left(2 \lambda_{n}\right)$. Because of (1) and the nature of $\lambda_{n}$, any possible $N_{2}$ will contain infinitely many $n_{2}$ associated with each possible value of $\lambda$. Thus $N_{2}$ splits into disjoint infinite sequences $M_{p}, p \geqq 1$, such that

$$
\lambda_{m_{p}}=p, \quad m_{p} \in M_{p} .
$$

For a given $h>0$, choose $p$ such that $\pi / p \leqq h$. Then

$$
\begin{aligned}
\int_{0}^{h}\left|f_{m_{p}}^{\prime}(x)\right| d x & >\int_{\pi /(2 p)}^{\pi / p}\left|f_{m_{p}}^{\prime}(x)\right| d x \\
& =\epsilon_{m_{p}} \mu_{m_{p}} \int_{\pi /(2 p)}^{\pi / p}\left|\sin \mu_{m_{p}}\left(x-\frac{\pi}{2 p}\right)\right| d x \\
& =\epsilon_{m_{p}} \int_{0}^{\pi \mu_{m p} /(2 p)}|\sin t| d t \geqq \epsilon_{m_{p}}\left[\mu_{m} / p\right],
\end{aligned}
$$

and (2) follows from this inequality and (3).

Finally we note that Dvoretzky's use of the word "clearly" in the third line after equation (6) is dubious.

\section{REFERENCES}

1. A. Dvoretzky, On a theorem of Walsh, Proc. Amer. Math. Soc. vol. 7 (1956) pp. 363-366.

2. J. L. Walsh, On continuity properties of derivatives of sequences of functions, Proc. Amer. Math. Soc. vol. 4 (1953) pp. 69-75.

The Rice Institute 